\title{
Effects of tele-rehabilitation mental practice on balancing performance of patients with hemiplegia
}

\author{
So Yeon Kim, Su Jin Park, Ye Eun Lee, Eun Ha Lee, Hwa Yeong Lim, In Gyu Yoo \\ Department of Occupational Therapy, Jeonju University, Jeonju, Korea
}

\section{Summary}

Study aim: The purpose of this study was to confirm the effectiveness of tele-rehabilitation mental practice (TRMP) on balancing performance for patients with hemiplegia.

Material and methods: This study included five stroke patients, and all participants were trained to help to recover balancing performance. TRMP was applied for a total of 15 training sessions for 3 weeks, and pre- and post-intervention evaluations were carried out during this study. The Berg Balance Scale was used to confirm the balance ability, the Modified Falls Efficacy Scale to confirm the falls efficacy, and the Wii program to confirm the right and left weight distribution ratio.

Results: As a result of this study, we could not confirm a significant difference between pre- and post-intervention balance capacity and fall efficacy. However, weight distribution showed a significant difference after the intervention.

Conclusion: TRMP may be applied in novel balance training for stroke patients.

\section{Keywords: Balance capacity - Falls efficacy - Hemiplegia - TRMP}

\section{Introduction}

Stroke patients suffer from various movement disorders. Palsy in particular is one of the problems disturbing the activities of daily living after stroke. Hemiplegia, one of the typical symptoms, causes an asymmetric posture, thus losing the ability to evenly distribute body weight, resulting in serious problems with balance. Generally, these problems can lead to inappropriately balance conditions, which exposes them to the dangerous situation of falling. The association of loss of balance with falls has been well documented and can lead to a decrease in physical activity and efficacy due to psychological fear, even if there is no past experience of falls $[2,17]$.

As the problem of falls is of social interest, various intervention methods have been studied to prevent it. The most common intervention methods that have been used in the past include the prevention of falling through education, the method of eliminating the risk factors of falling through home modification, and the prevention of falling through exercise programs for improving physical ability. The most commonly applied method has been fall prevention through an exercise program. However, it is not easy for exercise programs to apply sustained intervention to patients. Many experts have sought to develop an exercise program that not only allows the subject to be more active, but also reduces the physical burden to improve these problems [24].

One method that has recently been introduced as an ideal intervention for patients is mental practice. Mental practice began with the theory that if you experience a step-by-step process of imagining a situation in the absence of any special movements, you can have a similar effect to the actual movement. This theory has been proven by past neuroscientific studies, and it has become even more interesting to demonstrate that the same part of the brain is activated during the actual task and through mental practice. In particular, this method is not only easy to apply, because it is not costly and does not require equipment, but it also has the advantage of being available anywhere in the home or hospital. However, some limitations of the studies were reported. In particular, we cannot confirm that they were properly focused during practice, and therapists must be in the same space for practice $[3,13]$.

Tele-rehabilitation is a novel approach for providing medical rehabilitation services by using information and communication technology to provide remote rehabilitation services. The intervention method has been recently described as an important factor in community-based rehabilitation because it has the advantage of being able to provide rehabilitation even if the patients do not visit the 
medical institution directly [22]. In the past, research on tele-rehabilitation, using various devices, com munication devices, and virtual reality devices, has been performed, and has demonstrated its therapeutic effectiveness. However, some limitations of the studies were reported. Park et al. (2008) proposed remote rehabilitation by telephone to compensate for these disadvantages. In the past, it had the limitation of communication according to the presence or absence of the user at a fixed telephone. However, recently, the mobile phone has minimized such disadvantages [8].

The results of previous studies show that the mental practice of elderly people aged 70 or older showed a significant improvement in balance ability. Six-week mental practice was shown to increase balance ability of elderly people in the community, and to improve balance components [3]. In addition, other research has shown that participants who are experiencing stroke have improved limb mobility, balance, and activities of daily living when telerehabilitation is applied [8]. However, previous research that confirmed the synergy of the two intervention methods was insufficient. Therefore, in this study, we want to confirm the effectiveness of application of TRMP to minimize the limitations of mental practice and tele-rehabilitation in balance capacity and falls efficacy of patients with hemiplegia.

\section{Material and methods}

This study was conducted from November 13th to 27th, 2016 at the J Public Health Center with prior consent. All the participants were informed of the study and gave consent. The participants were 7 stroke patients, but 2 patients dropped out, and finally, 5 patients participated. They received an explanation about the purpose and methods of the study and provided informed consent prior to participation in accordance with the ethical principles of the Declaration of Helsinki.

\section{1) Inclusion criteria}

- MMSE-K score of 24 or more;

- Stroke duration of 6 months or more due to hemiplegia;
- VMIQ score of 2.26 or less;

- Using a mobile phone;

- Without hearing impairment;

- Communicable;

- Have never undergone mental practice;

- Can stand for one minute without assistive device $[8,13]$.

\section{2) Instruments}

Mini-Mental State Examination Korean, MMSE-K

The MMSE, developed by Folstein (1975), is a tool for the elderly in the community to easily measure the presence of cognitive impairment or dementia symptoms [6]. The evaluation scale consisted of 5 points for time, 5 points for location, 3 points for memory registration, 3 points for memory recall, 5 points for attention and calculation, 7 points for language function, and 2 points for understanding and judgment. The evaluation results are defined as 24 points or more as normal, 18-23 as mild cognitive dysfunction, and 17 or less as severe disability. The inter-tester reliability of this instrument was very high (0.86) [16], and this study was used for cognitive ability assessment to determine whether the cognitive abilities of imaginative training were selected.

\section{Vividness of Movement Imagery Questionnaire, VMIQ}

It is composed of 24 items as a tool for assessing imagination ability, developed by Issac et al. (1986) [10]. Each item measured imagination ability, which could specify body movement in imagery items (on a scale from 1 point (no image at all) to 5 points (perfectly clear)). The testretest reliability was 0.76 and the validity was very high (0.81) with the imaginary test (VMIQ). Our study was used to select those with a mean VMIQ score of 2.26 or less.

\section{Berg Balance Scale, BBS}

The Berg Balance Test was developed by Berg et al. In 1989 and is used for balance assessment regardless of age [1]. It is also used to evaluate the ability of the stroke patient to walk, to determine whether to use assistive devices, and to predict the possibility of independent walking. There are 14 evaluation items in total. The scoring

Table 1. Characteristic of participants

\begin{tabular}{|c|c|c|c|c|c|}
\hline Participants & Sex & Age & Affected side & MMSE-K & VMIQ \\
\hline Participant 1 & Female & 72 & Right & 25 & 1.79 \\
\hline Participant 2 & Female & 71 & Left & 27 & 2.25 \\
\hline Participant 3 & Male & 65 & Right & 27 & 1.62 \\
\hline Participant 4 & Male & 64 & Left & 30 & 1.87 \\
\hline Participant 5 & Male & 53 & Right & 27 & 1.00 \\
\hline
\end{tabular}


system consists of a scale of $0-4$ points and a total score of 56 points. The inter-tester reliability of the tool was 0.97 and the intra-tester reliability was 0.96 [11]. This study was used to evaluate the balance ability of hemiplegic patients.

\section{The Modified Falls Efficacy Scale, MFES}

The fall efficacy scale questionnaire is widely used to assess the self-perception of falls in older people [9]. The fall efficacy scale questionnaire consists of 14 items, consisting of a $0-10$ point scale, and the total score of the test is 140 points. 0 means that fall efficacy is the lowest, and 140 points means that fall self-efficacy is the highest, which means that self-confidence is the highest. The reliability of this instrument is very high, with Cronbach's alpha $=0.971$ [20]. This study was used to evaluate the fall efficacy of hemiplegic participants.

\section{Nintendo Wii Program}

Nintendo's Wii Balance Board is a home-use game console that is relatively inexpensive and has a pressure sensor mounted on each corner of the square to measure weight distribution and COP (center of pressure). It is easy to use. The test-retest reliability of the balance board was ICC $=0.66$ to 0.94 [4]. The test-retest reliability of the Balancia program, which can measure the ratio of the right and left weight distribution, was reported as $\mathrm{ICC}=0.79$ [21]. This study was used to measure left and right weight distribution of hemiplegic participants.

\section{3) Research design}

In this study, one-group pretest-posttest design was used to determine the effects of distance imagination training on balance capacity, fall efficacy, and right and left weight distribution ratios for five participants. In this study, the researcher trained by using the smart phone for the application of the remote mental practice intervention to the participant. The training started with closed eyes while sitting comfortably in the living environment. The training program consisted of three sessions, including a one-time course (10 minutes) under the supervision of the researcher and two sessions of self-imagination training (two 5-minute sessions) using the recorded voice file. Because of the nature of remote imaginary training, it is difficult to identify whether the participants are concentrating, so that they can continue to focus on training by using the question method applied in previous research [12].

\section{4) Data analysis}

Descriptive statistics were used to analyze the statistical differences of the evaluated data in the participants, and the Wilcoxon signed rank test was used to test for significance before and after the intervention. Statistical analysis was performed with SPSS 22.0 and statistical significance was defined as $\mathrm{p}<0.05$.

\section{Results}

As a result of this study, we could not confirm a significant difference between pre- and post-intervention balance capacity and fall efficacy. However, weight distribution showed a significant difference after the intervention. In fact, balance capacity and fall efficacy also showed positive improvements in the results. In the case of the BBS, the score of participant 2 increased from 50 to 52, the score of participant 4 from 38 to 40, and the score of participant 5 from 37 to 43 . In the case of the MFES, the score of participant 1 improved from 8.21 to 9.42 , the score of participant 2 from 3.28 to 7.21 , the score of participant 4 from 5.07 to 5.92 , and the score of participant 5 from 3.92 to 5.60. In the case of the weight distribution chart, participant 1 ranged from 3.60 to 1.60 , participant 2 from 24.20 to 16.00 , participant 3 from 5.00 to 4.00 , participant 4 from 10.80 to 4.00 , and participant 5 from 29.20 to 28.00 , indicating a decrease in the ratio of weight distribution.

\section{Discussion}

The purpose of this study was to investigate the change of balancing performance of hemiplegic patients using the TRMP. After applying the TRMP to the participants, there was no statistically significant difference in the balance

Table 2. Comparison of balancing performance in participations

\begin{tabular}{lccrr}
\hline & $\mathrm{M} \pm \mathrm{SD}$ & $\mathrm{z}$ & $\mathrm{p}$ \\
\hline & $\mathrm{Pre}$ & Post & & \\
\hline BBS & $46.80 \pm 8.70$ & $48.4 \pm 6.43$ & -1.134 & 0.257 \\
MFES & $5.23 \pm 1.91$ & $6.76 \pm 1.62$ & -1.826 & 0.068 \\
Wii program (Weight bearing ratio) & $13.56 \pm 10.08$ & $8.32 \pm 7.08$ & -2.032 & $0.042^{*}$ \\
\hline
\end{tabular}

$\mathrm{M}=$ mean, $\mathrm{SD}=$ standard deviation; $\mathrm{BBS}=$ Berg Balance Scale; MFES = Modified Falls Efficacy Scale. 


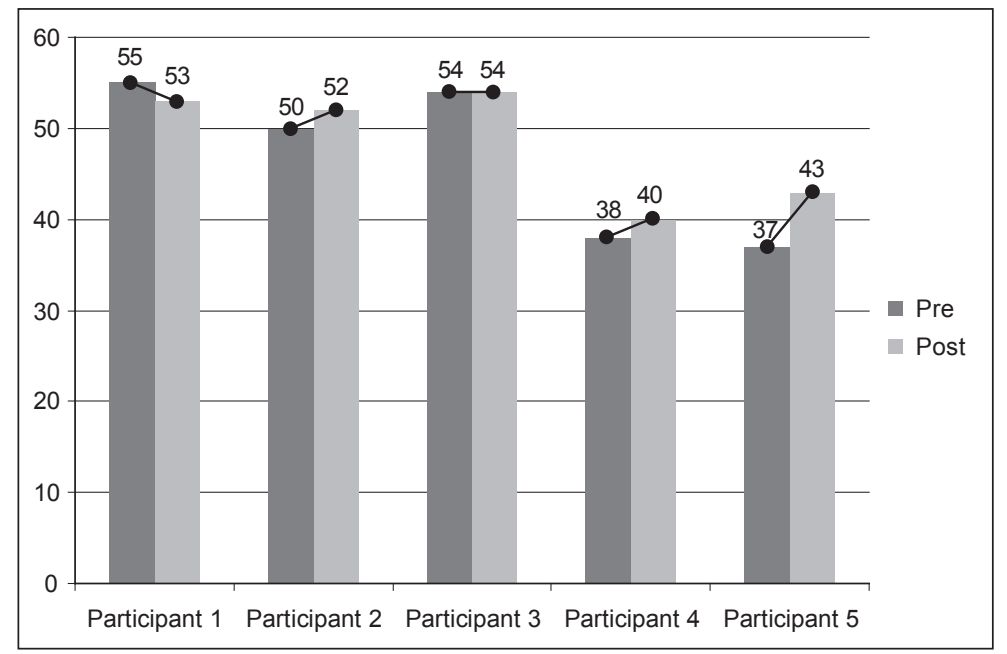

Fig. 1. Comparison of Berg Balance Scale in participants

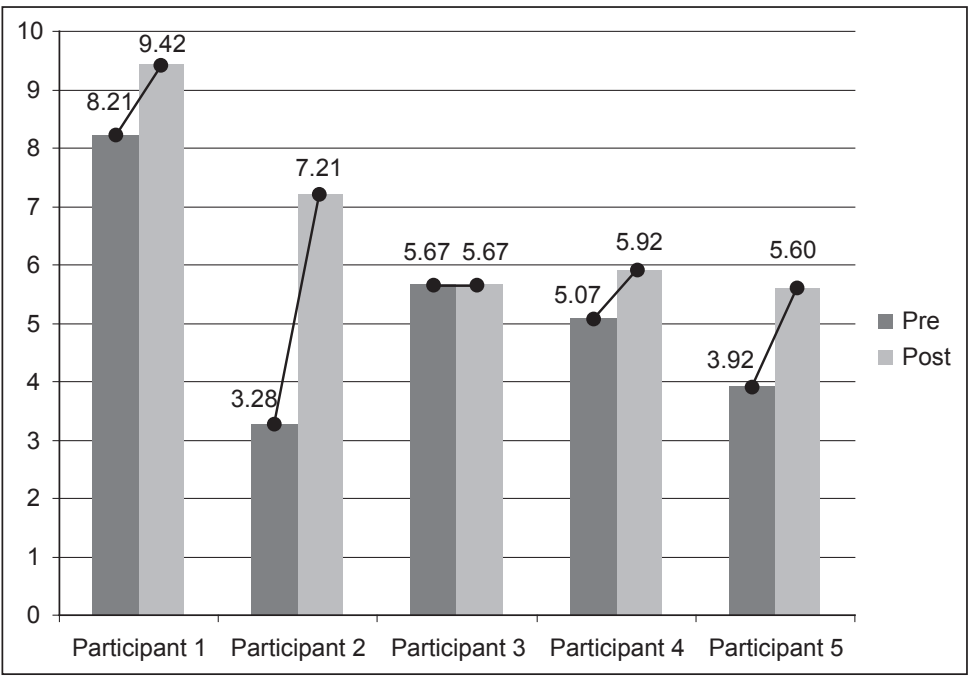

Fig. 2. Comparison of Modified Falls Efficiency Scale in participants

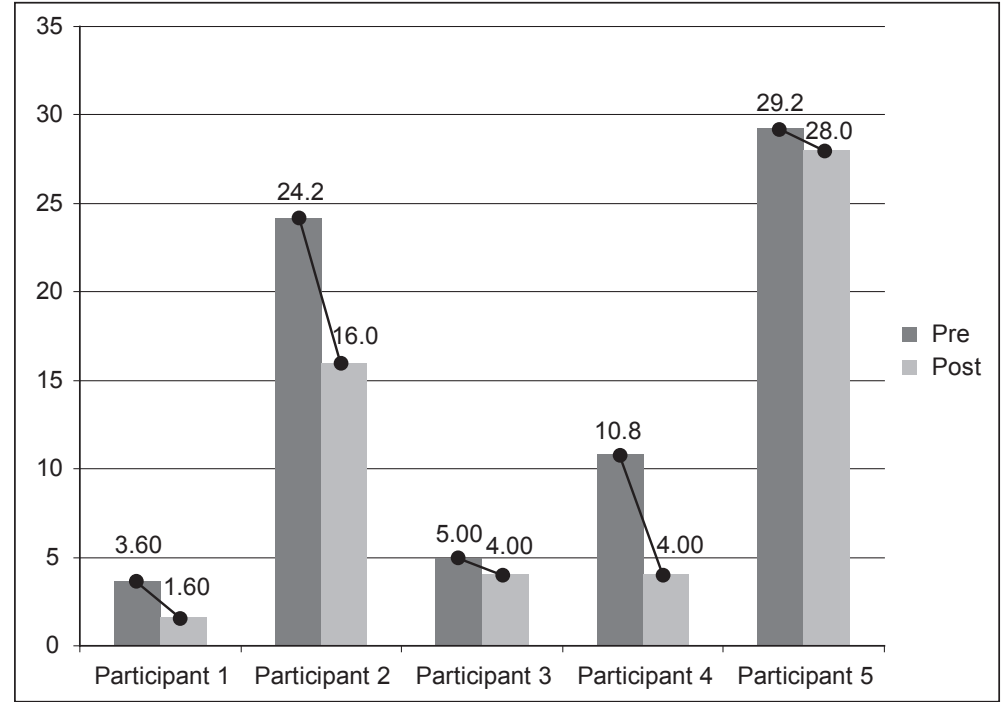

Fig. 3. Comparison of weight bearing ratio in participants 
capacity or fall efficacy. However, weight distribution showed a significant difference after the intervention. Although there was no statistically significant difference in the balance ability or fall efficacy, positive improvements were confirmed in the results.

This study had an advantage to find the effectiveness of the combined approach with mental practice and telerehabilitation. In the past, several studies using mental practice as an intervention method have explained the effectiveness of providing auditory feedback in the application of imagination training to the elderly, and they have had the disadvantage of not being able to confirm whether or not the participant maintains concentration due to the nature of imagination training. In order to overcome these drawbacks, we selected only participants with a VMIQ score of 2.26 or less and an MMSE-K score of 24 points or more, and we were able to concentrate on training as much as possible during questioning during the imagination exercise [23]. In the case of training time, 10 minutes per session was used based on the results of past research. Dickstein et al. (2004) applied 15 training sessions for 3 weeks [5].

The Berg Balance Scale (BBS) was used to identify changes in the participant's balance ability after the imaginary training. Participants 2, 4, and 5 improved by 6 , 2 , and 2 points, respectively. Participant 1 decreased by 2 points. The high baseline value of the time of onset and the BBS of the participants in this study may have influenced the results. In this study, the MFES was used to compare differences in fall efficacy. This tool has been proven to be reliable for predicting the risk of falling [19]. Five hemiplegic patients participating in this study were found to maintain and improve the fall efficacy scores after the intervention. Finally, weight distribution ratios were measured and compared before and after the intervention using the Nintendo Wii program to determine weight distribution while standing. All five participants showed a tendency to stabilize the weight center ratio. This is consistent with the finding that past mental practice can lead to activation of the nervous system related to proprioceptive and kinesthetic senses.

This study confirms that the application of TRMP to community chronic hemiplegic patients affects the balancing performance of the participants. In addition, the effect of TRMP may be enough to positively affect the balancing performance of hemiplegia, even though it was performed less frequently than in the previous studies. The intervention methods for fall prevention have mainly applied methods through education, environment modification, and exercise $[7,12,14,15]$. When compared with these intervention methods, TRMP can be applied to elderly people who have economic, time, and place restrictions as well as those for whom it is difficult to access rehabilitation service $[18,21]$. In particular, the TRMP method using the telephone applied in this study is very useful because it uses the easily accessible telephone rather than the application of expensive equipment and complicated operation, which was mentioned as a limitation in previous tele-rehabilitation. It is expected that this application of tele-rehabilitation will be effective for self-management and independence of daily living ability.

The limitation of this study was that it was difficult to select participants because there were many criteria such as MMSE-K (24 points or more) and VMIQ (2.26 or less). In addition, the results of this study showed that hemiplegic participants tended to find it difficult to imagine the motion during remote imaginary training, which made it difficult to train. Lastly, there was a limitation in that it was not possible to take into account differences in training application due to differences in intonation, attitude, and training application time of the trainers. Further research should be conducted to study the effect of distance imagery training, and to study the effect of long-term imagery training, and it is necessary to continue the study to prove the effect of remote imagination training by increasing the number of participants.

\section{Conflict of interest: Authors state no conflict of interest.}

\section{References}

1. Berg K., D.S. Wood, J.I. Williams, D. Gayton (1989) Measuring balance in the elderly : Preliminary development of an instument. Physiother. Can., 41(6): 304-311.

2. Cho S.I., D.H. An (2012) Effects of a fall prevention exercise program on muscle strenght and balance of the old-old elderly. J. Phys. Ther. Sci., 26(11): 1771-1774.

3. Chung J.C., H.Y. Jung, C.D. Lee, E.Y. Yoo (2015) Effects of mental practice on balance ability and fall efficacy in dwelling elderly population: Single subject design. J. Korean Soc. Commun. Based Occup. Ther., 5(2): 31-42.

4. Clark R.A., A.L. Bryant, Y. Pua, P. McCrory, K. Bennell, M. Hunt (2010) Validity and reliability of the nintendo will balance board for assessment of standing balance. Gait Posture, 31(3): 307-310.

5. Dickstein R., A. Dunsky, E. Marcovitz (2004) Motor imagery for gait rehabilitation in post-stroke hemiparesis. Phys. Ther., 84(12): 1167-1177.

6. Folstein M.F., S.E. Folstein, P.R. McHugh (1975) "Minimental state". A practical method for grading the cognitive state of patients for the clinician. J. Psychiatr. Res., 12(3): 189-198.

7. Freiberger E., L. Haberle, W.W. Spirduso, G.A. Zijlstra (2012) Long-term effects of three multicomponent exercise interventions on physical performance and fall-related psychological outcomes in community-dwelling older 
adults : A randomized controlled trial. J. Am. Geriatr. Soc., 60(3): 437-446.

8. Han S.H., D.I. Kim, J.H. Kim, C.H. Park, B.M. Lee, K.Y. Kam (2012) Effect of telerehabilitation using mobile phone on motor function recovery in chronic stroke patients. J. Korean Soc. Commun. Based Occup. Ther., 20(3): 67-79.

9. Hill K.D., J.A. Schwarz, A.J. Kalogeropoulos, S.J. Gibson (1996) Fear of falling revisited. Arch. Phys. Med. Rehabil., 77(10): 1025-1029.

10. Isaac A., D.F. Marks, D.G. Russell (1986) An instrument for assessing imagery of movement: The vividness of movement imagery questionnaire(VMIQ). J. Ment. Image, 10: 23-30.

11. Jung H.Y., J.H. Park, J.J. Shim, M.J. Kim, M.R. Hwang, S.H. Kim (2006) Reliability test of korean version of berg balane scale. J. Korean Acad. Rehabil. Med., 30(6): 611-618.

12. Kim J.S. (2012) Clinical guideline for the application of mental practice in neurological rehabilitation. Korean $J$. Neural. Rehabil., 2: 19-25.

13. Kim J.S., K. Kim (2010) Effect of motor imagery training with visual and kinesthetic imagery training with visual and kinesthetic imagery training on balance ability in post stroke hemiparesis. J. Korean Soc. Phys. Ther., 5(4): 517-525.

14. Kim M., J.S. Kim (2013) Effects of the fall prevention education program (FPEP) for caregivers in elderly care facilities on fall-related knowledge, fall-related burden, and caring behaviors for fall prevention. J. Korea Cont. Assoc., 13(11): 778-790.

15. Kim T.H., D.S. Oh (2000) Effects of exercise training on strength and balance for the elderly. Phys. Ther. Korea., 7(1): 32-37.

16. Lee C.S., S.C. Shin (1993) Standardization of the MiniMental State Examination (MMSE) in Korea. J. Korean Neuropsychiatr. Assoc., 32(6): 950-961.

17. Lee J.H., S.K. Kim, S.Y. Choi (2012) The effect of mental practice on increasing balance and fall index \& fall efficacy scale of stroke patients : Single subject experimental research. J. Korean Soc. Commun. Based Occup. Ther., 2(2): 37-49.
18. Lees F.D., P.G. Clark, C.R. Nigg, P. Newman (2005) Barriers to exercise behavior among older adults : A focusgroup study. J. Aging Phys. Act., 13(1): 23-33.

19. Park C.S., S.H. An (2013) A study on the clinical utility of the fall efficacy scale for predicting falls in stroke patients. J. Korean Soc. Occup. Ther., 21(1): 27-40.

20. Park G.B., B.R. Cho, I.S. Kwon, B.J. Park, T.K. Kim, G.Y. Cho, M.J. Kim (2010) Reliability and validity of korean version of falls efficacy scale-international(KFES-I). Ann. Rehabil. Med., 34(6): 554-559.

21. Park J.H. (2013) A study on effects of participation in social learning activities old elderly people in an agricultural village in self-efficacy and life satisfaction : Focusing on participants in a rural healthy and long life villages project (master's thesis), Chung-Ang university, Seoul.

22. Park Y.S., K.J. Han, Y.S Hah, M.S. Song, S.J. Kim, J.W. Chung (2008) Effects of telephone counseling on health and service satisfaction after discharge in gynecologic cancer women. J. Korean Acad. Soc. Nurs. Educ., 14(2): 294-304.

23. Yoo E.Y. (2000) The effect of mental practice along with visual feedback on symmetrical weight bearing training for persons with hemiparesis (doctoral dissertation), Yonsei University, Seoul.

24. Yoo E.Y., M.Y. Jung (2001) Mental practice and its potential for stroke rehabilitation. J. Koren Soc. Occup. Ther., 9(1): 153-161.

\section{Received 27.02.2018 \\ Accepted 18.06.2018}

(c) University of Physical Education, Warsaw, Poland

\section{Acknowledgments}

I would like to thank the co-authors who took the time to provide information for the study. We would like to thank the professors at the Department of Occupational Therapy in Jeonju University who provided insight and expertise that greatly assisted this study. 